\title{
Transmitter-receiver-transmitter configurations of ground-penetrating radar
}

\author{
Levent Gürel and Uğur Oğuz \\ Department of Electrical and Electronics Engineering, Bilkent University, Ankara, Turkey
}

Received 29 May 2001; revised 8 November 2001; accepted 9 November 2001; published 11 May 2002.

[1] Three-dimensional ground-penetrating radar (GPR) geometries are simulated using the finite difference time domain (FDTD) method. The GPR is modeled with a receiver and two transmitters with arbitrary polarizations in order to cancel the direct signals emitted by the two transmitters at the receiver. This GPR configuration is used to simulate scenarios involving single or multiple targets with arbitrary sizes. The buried objects are modeled as cylindrical disks. Perfectly matched layer absorbing boundary conditions are used to terminate the layered FDTD computational domain. INDEX TERMS: 3210 Mathematical Geophysics: Modeling; 3230 Mathematical Geophysics: Numerical solutions; 6969 Radio Science: Remote sensing; 0644 Electromagnetics: Numerical methods; 0933 Exploration Geophysics: Remote sensing; KEYWORDS: ground-penetrating radar (GPR), finite difference time domain (FDTD) method, subsurface scattering, perfectly matched layer

\section{Introduction}

[2] The need for simulating ground-penetrating radar (GPR) systems [Daniels, 1996; Moghaddam et al., 1991; Bourgeois and Smith, 1996] has increased the popularity of the finite difference time domain (FDTD) method [Yee, 1966] among other numerical modeling techniques. The FDTD method is a powerful tool in solving problems involving layered media and complicated inhomogeneities [Moghaddam et al., 1991; Gürel and $\mathrm{Ogguz}_{\mathrm{g}}$ 2001]. In this paper, three-dimensional GPR scenarios are simulated using the FDTD method and the perfectly matched layer (PML) [Berenger, 1994; Chew and Weedon, 1994] absorbing boundary conditions (ABC).

[3] The geometry of a GPR problem consists of two half-spaces, the air modeled as a vacuum and the ground modeled as a homogeneous dielectric medium, separated by an interface, as shown in Figure 1. The ground can also be modeled as a lossy and heterogeneous medium. The simulation results of such ground models are presented in other reports [Gürel and Ŏguz, 2001; Oğuz and Gürel, 2002; L. Gürel and U. Oğuz, Transmitter-receiver-transmitter-configured ground-penetrating radars over randomly heterogeneous ground models, submitted to Radio Science, 2001] (hereinafter

Copyright 2002 by the American Geophysical Union. 0048-6604/02/2001RS002500\$11.00 referred to as Gürel and Oğuz, submitted manuscript, 2001).

[4] Similar to the FDTD computational domain, the PML regions are also designed as two layers, matching both the ground and air regions and the interface between them. Buried targets can be modeled with arbitrary quantity, shapes, permittivities, and conductivities. The radar unit contains the transmitting and receiving antennas. These antennas move over the ground-air interface at a fixed elevation, as depicted in Figure 1.

[5] In this paper, buried targets are modeled as single or multiple conducting disks. A realistic scenario involving two nonidentical disks is also simulated. Other scenarios involving similar GPR configurations but different ground and target features, such as dielectric and conducting targets of rectangular prism shape and ground models with higher permittivities, are reported by Gürel and $O \check{g} u z$ [2000].

\section{Radar Unit}

[6] Most of the GPR models found in the literature exhibit a transmitter-receiver (TR) configuration to illuminate the target and collect the scattered fields [Moghaddam et al., 1991; Bourgeois and Smith, 1996]. In that configuration the total signal contains the sum of the desired scattered signal $S$, the direct signal $D$, and the signal reflected from the ground $G[O \check{g} u z$ and Gürel, 2001]. Usually, the total collected signal is dominated by 


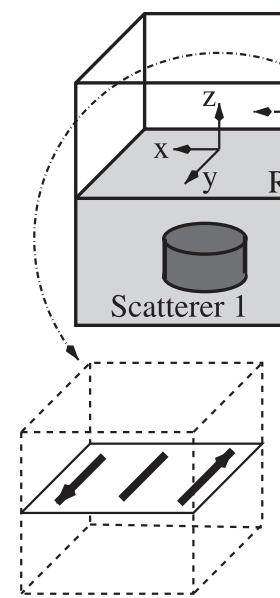

GPR2
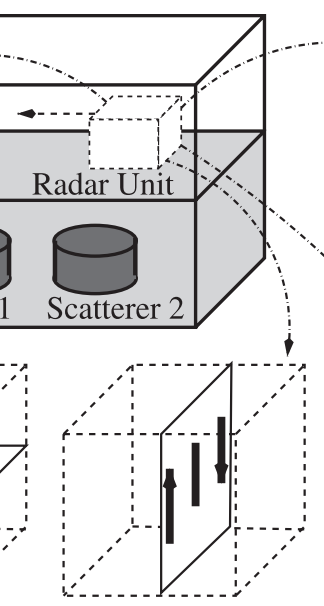

GPR3

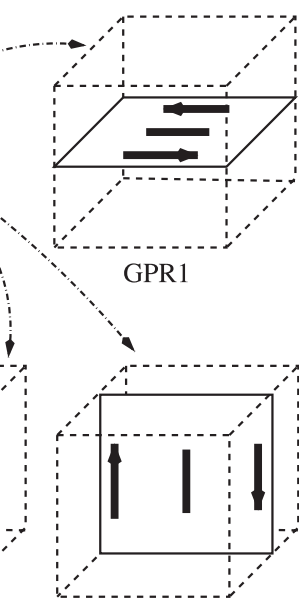

GPR4
Figure 1. Geometry of a half-space problem with a buried scatterer and the GPR models used in this work: GPR1 is configured as three $x$-polarized antennas aligned in the $y$ direction. GPR2 consists of three $y$-polarized antennas aligned in the $x$ direction. GPR3 and GPR4 represent three $z$-polarized antennas aligned in the $y$ and $x$ directions, respectively.

the $D$ signal, and it is either hard or impossible to detect the desired $S$ signal in the total received signal. Additional hardware and software components are developed in order to facilitate the detection of the target signal in the large background signal (U. Oğuz and L. Gürel, Electromagnetic simulation of various techniques to facilitate detection in ground-penetrating-radar problems, submitted to Geophysics, 2001) or to reduce the amplitudes of the unwanted signals [Ŏguz and Gürel, 2001; Gürel and Ŏguz, 1999; Bourgeois and Smith, 1998].

[7] In this work, radar units consist of two transmitters $\left(T_{1}\right.$ and $\left.T_{2}\right)$ and a receiver $(R)$, as shown in Figure 2. The two transmitters are fed with a phase difference of $180^{\circ}$. In this configuration the two direct signals $D_{1}$ and $D_{2}$ cancel each other everywhere on a symmetry plane that is equidistant to the two transmitters. The location of the $\mathrm{R}$ antenna is chosen to be exactly in the middle of two transmitters, coinciding with the symmetry plane [Luneau and Delisle, 1996; Gürel and Ŏguz, 2000]. Similarly, the two reflected signals $G_{1}$ and $G_{2}$ also cancel out at the receiver, if the ground is homogeneous. Consequently, in the transmitter-receiver-transmitter (TRT) configuration, the total received signal is solely due to the buried object $\left(S_{1}+S_{2}\right)$, which leads to the detection of the buried object. The cases of inhomogeneous grounds are studied elsewhere (Gürel and Oğuz, submitted manuscript, 2001).
[8] Figure 1 displays four GPR models, referred to as GPR1, GPR2, GPR3, and GPR4. The four TRT configurations differ from each other in the polarization and alignment of the three antennas [Gürel and Oğuz, 2000, 2002]. Each transmitting antenna is modeled by a singlecell $(\Delta)$ volume current density. The time variation of the current source is given by

$$
J(t)=\frac{1}{\Delta^{3}}\left[4\left(\frac{t}{\tau}\right)^{3}-\left(\frac{t}{\tau}\right)^{4}\right] e^{-t / \tau},
$$

where $\tau=1 /\left(4 \pi f_{0}\right)$ and $f_{0}$ is the center frequency of the pulse. The receiver is selected as a single-cell dipole that samples and stores the values of the $x, y$, or $z$ component of the electric field $E^{n}$. The data collected at a single point for successive instants of time $n$ are called an A scan. When the GPR unit moves on a linear path and performs A-scan measurements at discrete points, the collection of these A-scan measurements is called a B scan.

\section{Simulation Results}

[9] In this section, simulation results of the four GPR models introduced in section 2 are presented. A center frequency of $f_{0}=1 \mathrm{GHz}$ is used in all of these simulations, and $\Delta=2.5 \mathrm{~mm}$ and $\Delta t=4.5 \mathrm{ps}$ are the sampling intervals in space and time, respectively. The transmitting and receiving antennas are separated by two cells $(2 \Delta)$.

\subsection{Single Conducting Disk}

[10] The four GPR models are first tested on a scenario involving a perfectly conducting disk with a radius of 10

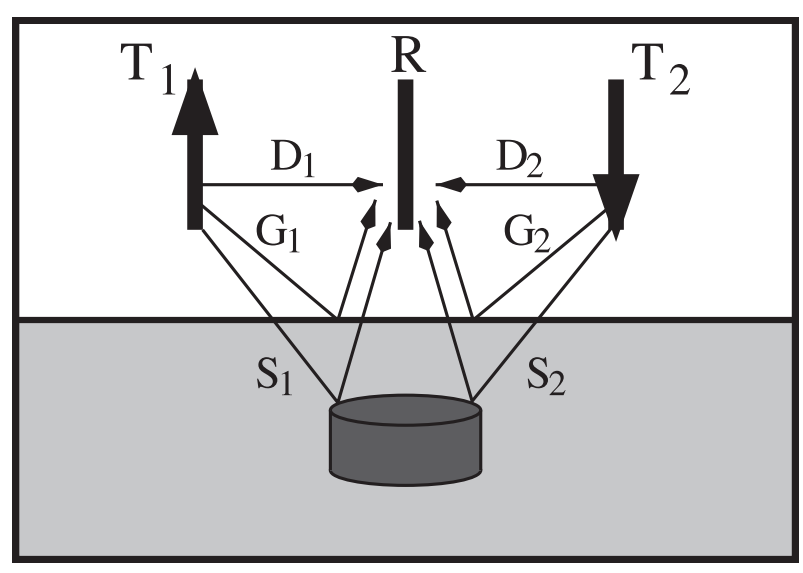

Figure 2. Transmitter-receiver-transmitter (TRT) configuration of the radar unit and the description of the direct $(D)$, reflected $(G)$, and scattered $(S)$ signals. 

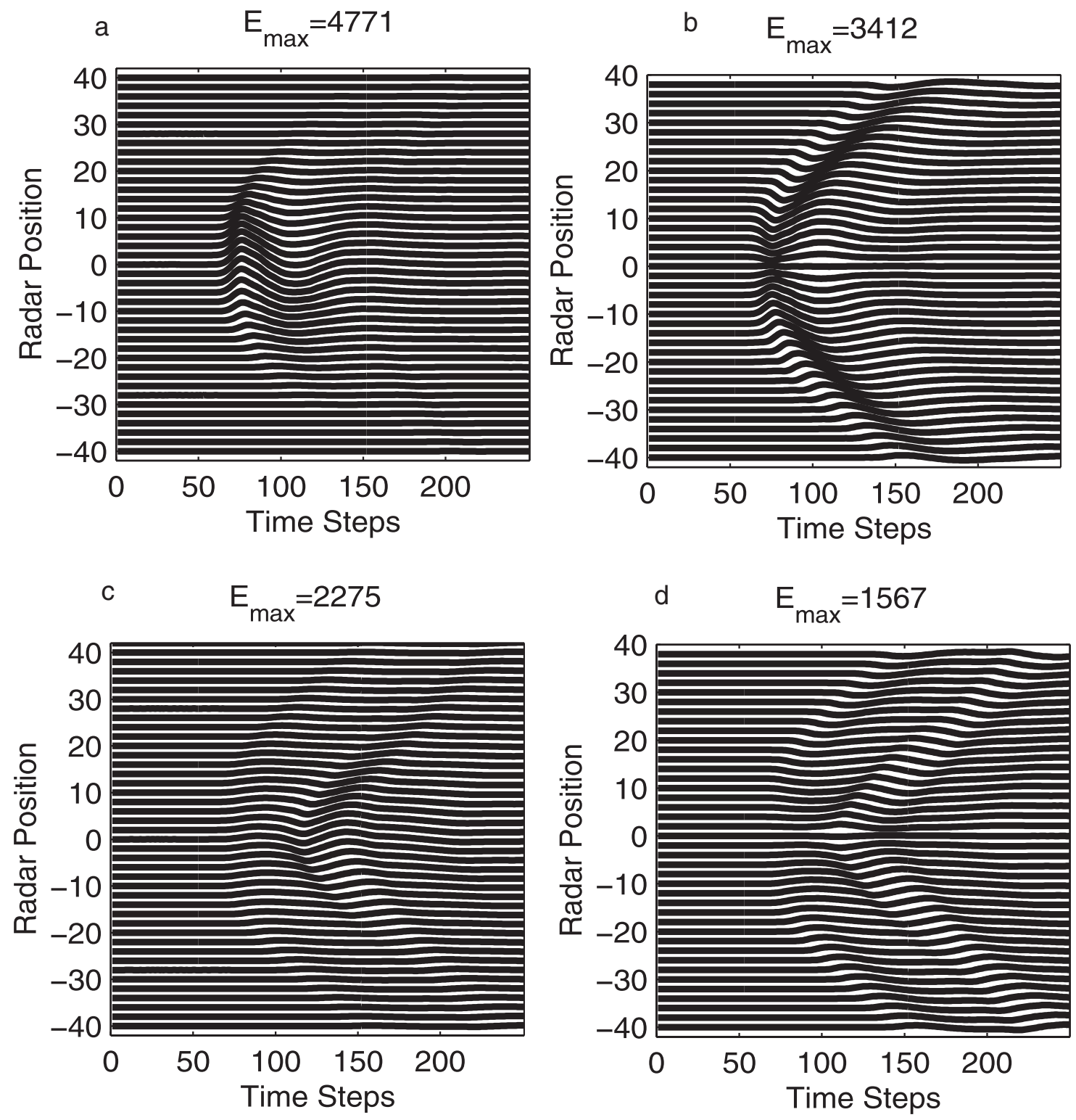

Figure 3. Simulation results of a perfectly conducting disk buried 5 cells under the ground. The simulations are carried out using (a) GPR1, (b) GPR2, (c) GPR3, and (d) GPR4.

cells buried 5 cells under the ground-air interface. The relative permittivity of the ground is selected as $\epsilon_{r}=2$. The radar unit travels on a straight line 10 cells away from the center of the disk, which corresponds to a path passing through the edge of the conducting disk. Figures $3 a-3 d$ present the B-scan results as a function of the radar position (vertical axis) and time (horizontal axis). For each GPR model the maximum value obtained in the B scan is given at the top of the corresponding plot. The largest of these four $E_{\max }$ values is used to normalize all four B-scan plots. Figures $3 \mathrm{a}-3 \mathrm{~d}$ show that the responses of the four GPR models are different even for the same scenario. GPR1 collects electric fields with the highest magnitudes. However, GPR1 produces visible responses only when the radar unit is very close to the target, while GPR2 responds even when the radar unit is far from the target.

[11] In order to further illustrate the differences in their responses the four GPR models are moved on a twodimensional grid, where an A-scan measurement is 

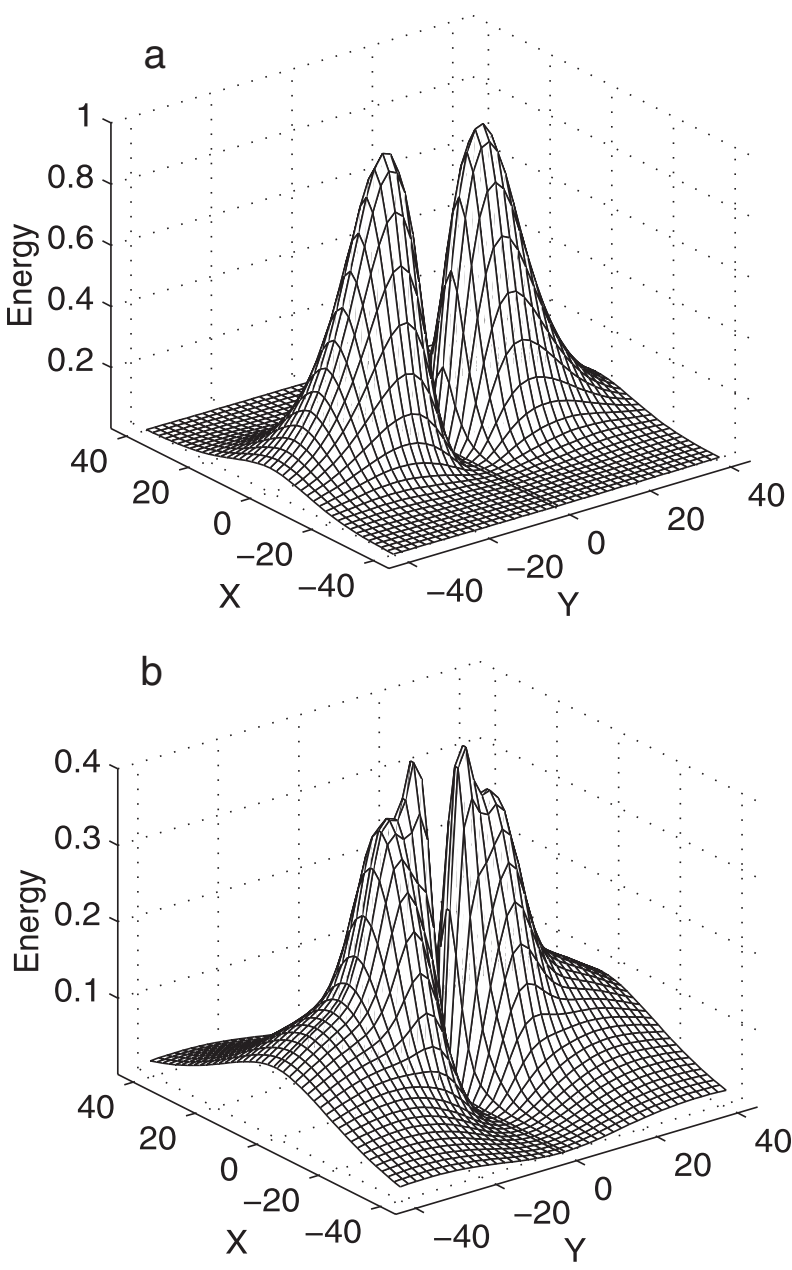

Figure 4. Energy diagrams measured by (a) $x$-polarized and (b) $z$-polarized TRT radar units moving on a two-dimensional grid. A perfectly conducting disk is buried 5 cells under the ground.

performed in each discrete position. The energy values of these A-scan signals are computed as

$$
\mathrm{ENERGY}=\sum_{n}\left|E^{n}\right|^{2}
$$

and given in Figure 4. Figures $4 \mathrm{a}$ and $4 \mathrm{~b}$ are obtained by radar units consisting of $x$ - and $z$-polarized dipoles, respectively. Since the radar units move in two directions, results obtained by the $x$-polarized configuration, displayed in Figure 4a, contain both GPR1 and GPR2 results. Similarly, the energy plot of the $z$-polarized configuration given in Figure $4 \mathrm{~b}$ encompasses both GPR3 and GPR4 models. Constant- $y$ and constant- $x$ traces taken from the two-dimensional grid of Figure $4 \mathrm{a}$ correspond to the energy plots of the waveforms obtained by GPR1 and GPR2, respectively. Similarly, Figure $4 \mathrm{~b}$ can be obtained by either GPR3 units moving in the $x$ direction or GPR4 units moving in the $y$ direction.

[12] Figures $4 \mathrm{a}$ and $4 \mathrm{~b}$ demonstrate that GPR 1 and GPR2 collect 2 times larger scattered energy than GPR3 and GPR4. A constant-y trace in Figure 4a, which corresponds to a GPR1 B scan, contains a single peak, located close to the center of mass of the target. However, a constant- $x$ trace, which is a B scan performed by GPR2, displays two peaks, located above the edges of the disk. A similar comparison can be made upon the investigation of the constant- $y$ and constant- $x$ traces of Figure $4 \mathrm{~b}$, which are the B-scan results obtained with GPR3 and GPR4, respectively. Therefore it is possible to conclude that GPR2 and GPR4 detect the edges of the disk, whereas GPR1 and GPR3 respond to the whole mass of the buried target.

[13] Figure 4a further demonstrates that GPR2 receives a detectable amount of energy while the radar unit moves away from the scatterer, if the path itself is close to the buried target. If the path is not close to the scatterer, the energy collected by GPR2 is ignorable everywhere on the path. In contrast, GPR1 responds only when it is close to the buried target, but these responses are detectable even if the path itself is away from the target.

\subsection{Multiple Conducting Disks}

[14] The detection of two closely buried disks (Figure 5) is investigated next, after having demonstrated the sensitivities of GPR2 and GPR4 to distant targets and GPR1 and GPR3 to nearby targets in section 3.1. Figure 6 presents the simulation results of two conducting disks, each with a 10-cell radius and a 16-cell height, buried 5 cells under the ground and separated by 20 cells. The

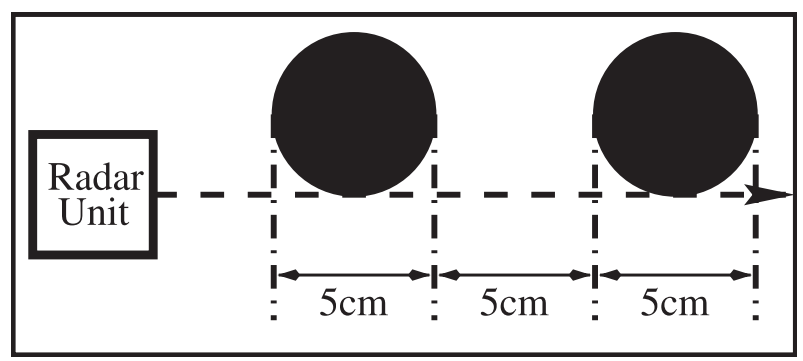

Figure 5. Simulation geometry that contains two identical perfectly conducting disks. Both disks have diameters of $5 \mathrm{~cm}$. The radar unit travels along a linear path that is tangential to both disks. 

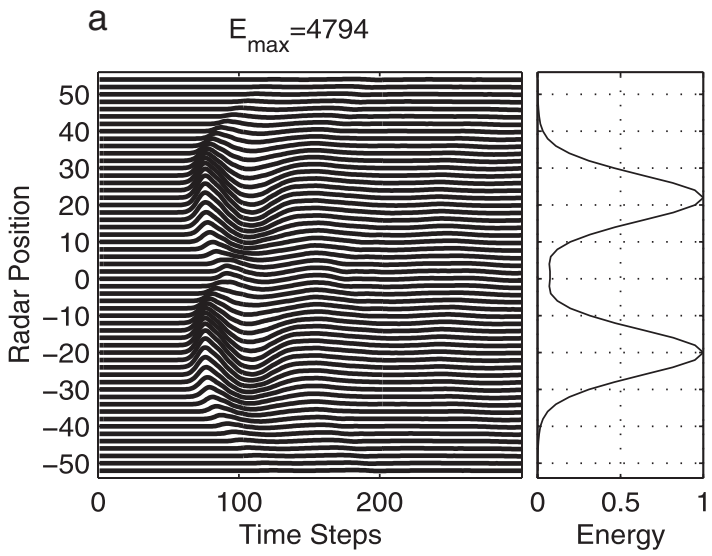

$\mathrm{C}$

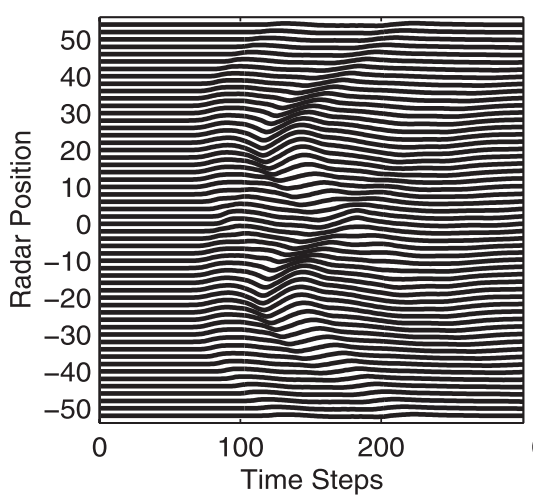

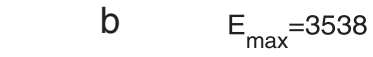
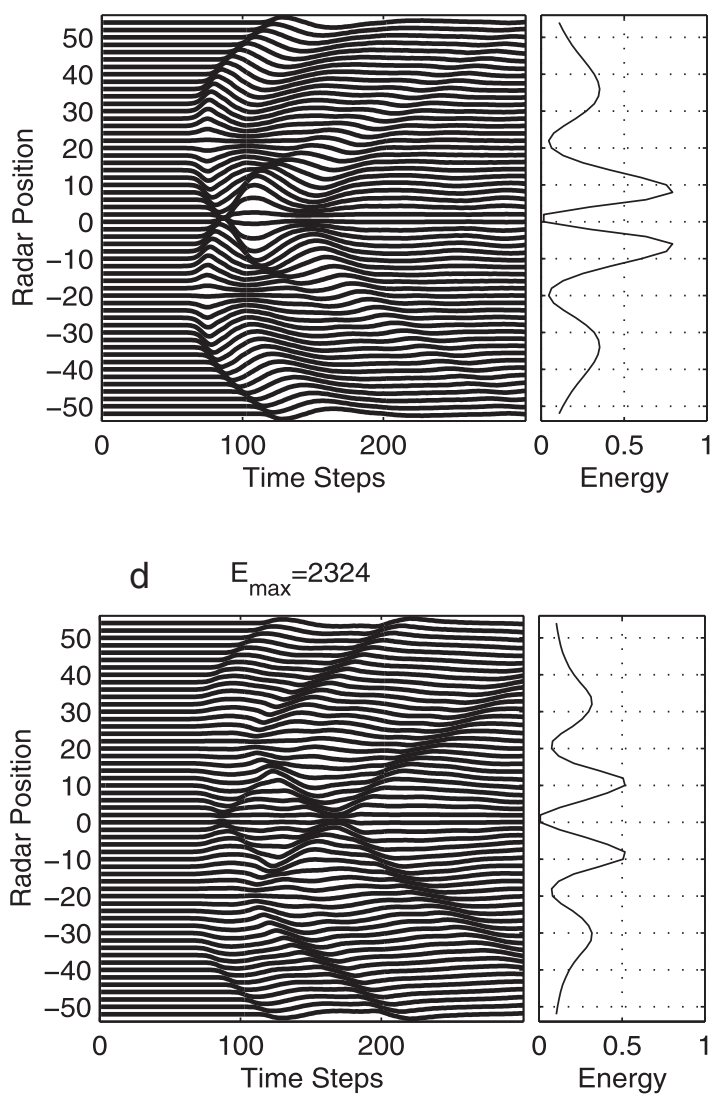

Figure 6. Simulation results of two identical perfectly conducting disks buried 5 cells under the ground and separated by 20 cells. Both disks have a diameter and height of $5 \mathrm{~cm}$ and $4 \mathrm{~cm}$, respectively. The ground has a relative permittivity of $\epsilon_{\mathrm{r}}=2$. The simulations are carried out using (a) GPR1, (b) GPR2, (c) GPR3, and (d) GPR4.

radar unit travels along a straight line above the ground. The projection of this path is tangent to both of the disks buried under the ground, as displayed in Figure 5. The relative permittivity of the ground is selected as $\epsilon_{r}=2$. The energies of the A-scan signals are evaluated according to equation (2) and presented in addition to the B-scan results in Figures $6 \mathrm{a}-6 \mathrm{~d}$. Figures $6 \mathrm{a}$ and $6 \mathrm{c}$ demonstrate that both objects can be detected by GPR1 and GPR3. However, the signals produced by GPR2 and GPR4 are not easy to interpret. Figures $6 \mathrm{~b}$ and $6 \mathrm{~d}$ contain two nulls, due to the minima encountered above the centers of mass of the two disks, as they pass near the two targets. However, a third null exists, which corresponds to the symmetry plane that is located exactly in the middle of the two objects. For this reason, Figures $6 \mathrm{~b}$ and $6 \mathrm{~d}$ do not clearly indicate the two targets under the ground.

[15] In Figure 5 the problem geometry is perfectly symmetric with respect to the plane in the middle of the two disks. Therefore all the results presented in Figures $6 \mathrm{a}-6 \mathrm{~d}$ are symmetric around the middle plane. In order to investigate a more general situation where such a symmetry does not exist, another simulation, involving two disks with different radii, is considered. The first conducting disk has a radius of $2.5 \mathrm{~cm}$, while the other disk's radius is $4 \mathrm{~cm}$. Both disks are $4 \mathrm{~cm}$ high and buried $5 \mathrm{~cm}$ under the ground. The four GPR models travel along a path whose projection is tangential to both disks, as shown in Figure 7. Figures 8a, 8b, 8c, and 8d display the signals recorded by GPR1, GPR2, GPR3, and GPR4, respectively. Similar to Figure 6, next to each B-scan result in Figure 8, the energies observed in the A-scan measurements are also presented. Figures $8 \mathrm{a}-8 \mathrm{~d}$ demonstrate the effects of the geometrical differences of this problem, compared to the results in Figure 6. Figure 8a displays two peaks of energy with similar amplitudes, but different widths, received by GPR1. 


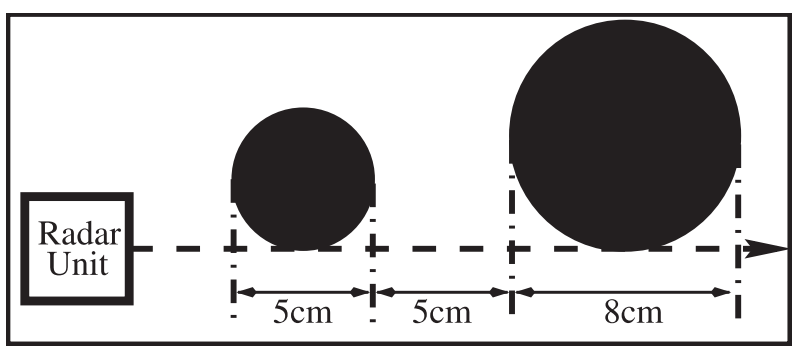

Figure 7. Simulation geometry that contains two nonidentical perfectly conducting disks, with $5 \mathrm{~cm}$ and $8 \mathrm{~cm}$ diameters. The radar unit travels along a linear path that is tangential to both disks.

GPR3 produces similar results, as demonstrated in Figure 8c. However, in Figure 8c, the maximum energy observed above the large disk is smaller compared to the energy observed above the small disk. In Figure $8 \mathrm{~b}$ the
B-scan measurements of GPR2 are shown. Although there is no symmetry plane between the two disks in this problem, GPR2 still encounters three minima. However, in Figure 8b, the minimum in the middle is not an exact null, in contrast to Figure 6b. The effects of the difference of the two disks are observed extensively in Figure 8d, where the results obtained by GPR4 are displayed. Similar to the GPR2 results in Figure 8b, Figure $8 \mathrm{~d}$ displays three minima in the B-scan measurements. Figures $8 \mathrm{~b}$ and $8 \mathrm{~d}$ demonstrate that it is not easy to detect the two closely placed disks with GPR2 or GPR4.

\section{Conclusion}

[16] Three-dimensional GPR scenarios are simulated using the FDTD method combined with the PML ABCs. The radar unit is modeled as a TRT configuration with
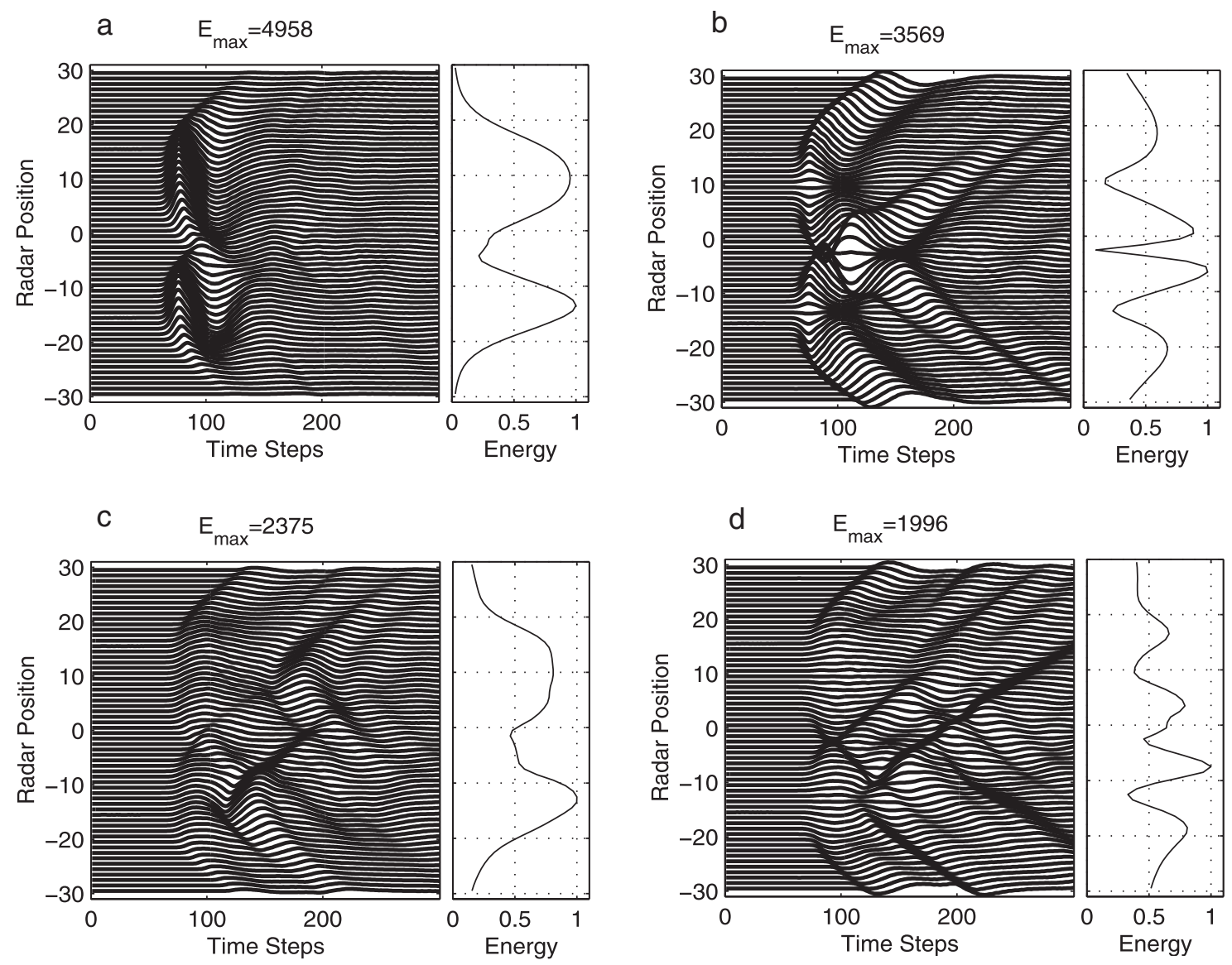

Figure 8. Simulation results of two nonidentical perfectly conducting disks buried 5 cells under the ground and separated by 20 cells. The heights of the disks are both $4 \mathrm{~cm}$. The diameters of the two disks are $5 \mathrm{~cm}$ and $8 \mathrm{~cm}$. The ground has a relative permittivity of $\epsilon_{\mathrm{r}}=2$. The simulations are carried out using (a) GPR1, (b) GPR2, (c) GPR3, and (d) GPR4. 
arbitrary polarizations of transmitting and receiving antennas. The targets are modeled as perfectly conducting cylindrical disks.

[17] The advantages of the TRT configuration are demonstrated using the simulation results. It is shown that the cancellations of the direct signals (due to the direct coupling from the transmitters to the receiver) and the reflected signals (from the ground-air interface) yield a total received signal that is only due to the scatterer, which facilitates the detection of the buried target. The specific advantages and disadvantages of various polarizations of the antennas in TRT configurations are demonstrated. The responses of the presented GPR models are different in character, which suggests that polarization-enriched GPR systems will achieve better detection performances.

[18] Acknowledgments. This work was supported by Bilkent University under Research Fund EE-01-01.

\section{References}

Berenger, J. P., A perfectly matched layer for the absorption of electromagnetic waves, J. Comput. Phys., 114, 185-200, 1994.

Bourgeois, J. M., and G. S. Smith, A fully three-dimensional simulation of a ground-penetrating radar: FDTD theory compared with experiment, IEEE Trans. Geosci. Remote Sens., 34(1), 36-44, 1996.

Bourgeois, J. M., and G. S. Smith, A complete electromagnetic simulation of the separated-aperture sensor for detecting buried land mines, IEEE Trans. Antennas Propag., 46(10), 1419-1426, 1998.

Chew, W. C., and W. H. Weedon, A 3D perfectly matched medium from modified Maxwell's equations with stretched coordinates, Microwave Opt. Technol. Lett., 7(13), 599604, 1994.
Daniels, D. J., Surface-Penetrating Radar, IEE, London, 1996.

Gürel, L., and U. Oğuz, Employing PML absorbers in the design and simulation of ground penetrating radars, paper presented at 1999 IEEE AP-S International Symposium and USNC/URSI National Radio Science Meeting, Inst. of Electr. and Electron. Eng., Orlando, Fla., July 1999.

Gürel, L., and U. Oğuz, Three-dimensional FDTD modeling of a ground-penetrating radar, IEEE Trans. Geosci. Remote Sens., 38(4), 1513-1521, 2000.

Gürel, L., and U. Oğuz, Simulations of ground-penetrating radars over lossy and heterogeneous grounds, IEEE Trans. Geosci. Remote Sens., 39(6), 1190-1197, 2001.

Gürel, L., and U. Oğuz, Optimization of the transmitter-receiver separation in the ground-penetrating radar, IEEE Trans. Antennas Propag., in press, 2002.

Luneau, P., and G. Y. Delisle, Underground target probing using FDTD, paper presented at 1996 IEEE AP-S International Symposium and URSI Radio Science Meeting, Inst. of Electr. and Electron. Eng., Baltimore, Md., July 1996.

Moghaddam, M., E. J. Yannakakis, W. C. Chew, and C. Randall, Modeling of the subsurface interface radar, J. Electromagn. Waves Appl., 5(1), 17-39, 1991.

Oğuz, U., and L. Gürel, Modeling of ground-penetrating-radar antennas with shields and simulated absorbers, IEEE Trans. Antennas Propag., 49(1), 1560-1567, 2001.

Oğuz, U., and L. Gürel, Frequency responses of ground-penetrating radars operating over highly lossy grounds, IEEE Trans. Geosci. Remote Sens., in press, 2002.

Yee, K. S., Numerical solution of initial boundary value problems involving Maxwell's equations in isotropic media, IEEE Trans. Antennas Propag., 14(4), 302-307, 1966.

L. Gürel and U. Oğuz, Department of Electrical and Electronics Engineering, Bilkent University, TR-06533, Bilkent, Ankara, Turkey. (lgurel@ee.bilkent.edu.tr; uoguz@ee.bilkent. edu.tr) 